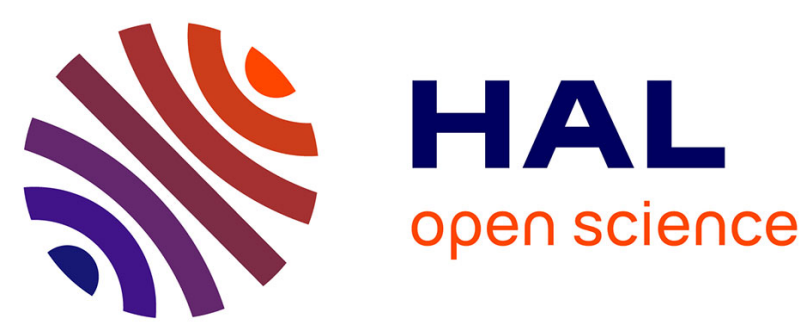

\title{
Colonialité, subjectivité, et déconstruction dans la pensée d'Édouard Glissant
}

Buata Malela

\section{To cite this version:}

Buata Malela. Colonialité, subjectivité, et déconstruction dans la pensée d'Édouard Glissant. Canadian Review of Comparative Literature/ Revue Canadienne de Littérature Comparée, 2016, 43 (3), pp.414-425. hal-02073354

HAL Id: hal-02073354

https://hal-normandie-univ.archives-ouvertes.fr/hal-02073354

Submitted on 4 Dec 2019

HAL is a multi-disciplinary open access archive for the deposit and dissemination of scientific research documents, whether they are published or not. The documents may come from teaching and research institutions in France or abroad, or from public or private research centers.
L'archive ouverte pluridisciplinaire HAL, est destinée au dépôt et à la diffusion de documents scientifiques de niveau recherche, publiés ou non, émanant des établissements d'enseignement et de recherche français ou étrangers, des laboratoires publics ou privés. 


\title{
Colonialité, SUbJECTIVITÉ, ET DÉCONSTRUCTION DANS LA PENSÉE D'ÉDOUARD GLISSANT
}

\author{
Buata B. Malela \\ Université de Mayotte
}

414 Cette étude examine un exemple paradigmatique d'une critique de la colonialité du pouvoir à partir d'une position intellectuelle qui vise à transcender la tradition nationale du fait intellectuel. L'écrivain et penseur originaire de Martinique, Édouard Glissant, nous servira de point de départ ; dès lors, il s'agira de montrer comment sont articulées et discutées les questions relatives à la colonialité (ou aliénation), à la subjectivité, à l'altérité dans son rapport à l'histoire des Antilles. Comment l'auteur de L'Intention poétique parvient à construire un discours littéraire et une pensée philosophique qui se posent en cadre alternatif et distinctif dans le monde intellectuel français et de la diaspora afrodescendante. On pourrait parler de "pensée alternative " parce qu'elle tend à s'écarter de la colonialité du pouvoir (ou l'aliénation) d'une part, et qu'elle s'insère dans une temporalité (ou moment) favorable, ce qui l'amène à modifier ce qui est déterminant dans le champ des discours sur les Antilles. Pour ce faire, Glissant propose ce qu'il appelle la "Relation " qui est un cadre large pour repenser la "globalisation. "

De là, l'hypothèse suivante : le discours alternatif en question proposé par Glissant tente de déconstruire l'eurocentrisme dans la manière de penser le rapport à la subjectivité (ou identité) et à l'aliénation. Celle-ci peut s'entendre comme une des puissances de la colonialité du pouvoir dont la force de structuration reste son internalisation par les agents, de sorte qu'elle leur apparaît comme une essence naturelle; ceux-ci seraient ainsi aliénés (Marx et Engels 319-20) parce que plus encore gouvernés par quelque chose qui puise son principe en dehors d'eux. Deux points forts appuient l'hypothèse susmentionnée. Primo, les données d'histoire de la pensée : l'écart par rapport à l'existentialisme et au structuralisme; secundo, la correspondance entre ces déterminations externes et la lecture de deux textes romanesques d'Édouard Glissant, à savoir Le Quatrième siècle (1964) et Malemort (1975), illustratifs de ce dis-

Canadian Review of Comparative Literature / Revue Canadienne de Littérature Comparée CRCL SEPTEMBER 2016 SEPTEMBRE RCLC

0319-051x/16/43.3/414 @ Canadian Comparative Literature Association 
cours alternatif à la pensée dominant les années 1960 et 1970 avec la mise en scène de figures de proximité qui semblent incarner l'expérience de la violence. Cette lecture par les expériences concrètes rejoint un des modes de pensée toujours actif dans un monde intellectuel peuplé par une génération de penseurs qui s'inscrivent bien en rupture avec d'autres moments de la pensée.

\section{RECONFIGURATION DU MONDE INTELLECTUEL}

La génération de penseurs, née entre 1920 et 1935, comme par exemple Michel Foucault, Gilles Deleuze, Jacques Derrida, et Édouard Glissant, tente une double rupture : rupture avec la phénoménologie de Sartre et Merleau-Ponty d'une part ; rupture avec le structuralisme de Lévi-Strauss d'autre part. Malgré la volonté de s'écarter de ces deux courants dominant la pensée d'après-guerre, ladite génération conserve les acquis de la critique marxienne des idéologies, de la généalogie nietzschéenne et de la psychanalyse freudienne (Besnier 642); elle renonce à l'idée de système directement inspirée de la pensée de Hegel qui, par le concept, ramènerait la totalité dispersée à une unité. Face à cette philosophie, Glissant propose de partir du Divers par exemple, d'où encore, le refus glissantien de ce qu'il appellera plus tard les "pensées de système " et les "systèmes de pensée. " Cette génération donne le primat à la forme (style), refuse les hiérarchies, le discours sur les identités et l'universalisme.

L'Écriture et la différence (1967) de Derrida reste emblématique à ce propos : la question de la différence y est une fenêtre ouverte sur l'opposition au structuralisme ambiant notamment. Au structuralisme, Derrida oppose la différence correspondant à la différence pure, c'est-à-dire en-soi et pour-soi, elle se rapporte à elle-même, en cela elle est sa propre négativité. L'identité est ainsi la différence. Et la différence reste ellemême différence et identité (Derrida 227-28). De la sorte, Derrida ouvre une réflexion portant sur l'altérité perçue comme identité et différence, montrant par-là la difficulté de penser au-delà de la raison que, par ailleurs, l'on prétend dénoncer. Il reste difficile de penser en dehors de l'historicité que l'on entend remplacer par une autre, un point que Glissant questionne dans ses différentes démarches à la fois en tant qu'écrivain et penseur. Or au centre de sa pensée et, contre la notion de système, Édouard Glissant place l'idée "d'expérience concrète " parce qu'elle lui permet de comprendre les problèmes de l'aliénation, de la subjectivité et de l'altérité en déconstruisant le discours antillais hérité des catégories de la domination.

La déconstruction de ces catégories de la domination nécessite de prendre conscience de la possibilité d'une autre histoire des Antilles, histoire dans laquelle l'expérience de l'esclavage s'incarne dans les figures de proximité. 


\section{LES FIGURES DE PROXIMITÉ}

Le récit glissantien se trouve traversé par les expériences de la souffrance, résultat de l'aliénation. Cette expérience fondamentale fonde les rapports du dominé à la complexité d'un univers peuplé de figures archétypales. Glissant les met en scène dans l'évocation de liens de parenté qui unissent et divisent les figures littéraires comme les Béluse et les Longoué dans le Quatrième siècle. Dans ce roman, le recours au verbe "engendrer" permet de dresser un discours catalogique sur les différentes figures des deux familles-celles-ci constituent les positions possibles dans la société esclavagiste-et permet au narrateur d'assurer une mémoire du passé, c'est-à-dire de l'histoire. Pour ce faire, ce récit pastiche le style biblique afin de montrer l'élection, parmi d'autres, de deux esclaves incarnant bien la souffrance et la subjectivité antillaise qu'essaie d'ailleurs de relater Papa Longoué, lui dont le fils est déjà mort, ce qui marque l'extinction de sa lignée :

Les Longoué ne pourraient plus veiller dans la forêt : la race allait s'éteindre. Car l'ancêtre avait engendré Melchior et Liberté le fils, et Melchior avait engendré Apostrophe et Liberté fille, et Apostrophe avait engendré Papa Longoué, et Papa Longoué avait engendré Ti-René, lequel avait engendré la mort subite. Mais ce n'était pas tant la mort. C'était qu'il fallait disposer d'un descendant, choisi, élu. Un jeune plant par lequel vous avez des racines dans la terre du futur. C'était cela. Se raccrocher à demain par les forces de la jeunesse. Mais Ti-René était mort trop vite. Il n’y avait plus que ce Mathieu-Béluse (Quatrième siècle 16).

Le lien de parenté permet aussi d'avancer dans "la terre du futur, " dans la pensée relative à une historicité antillaise reconstruite grâce aux "forces de la jeunesse. " Autrement dit, cette évocation amène Glissant à deviser différemment des Antilles contre la logique propre à la colonialité du pouvoir. De plus, en soulignant la fin de la fonction des Longoué en tant que quimboiseur-sorcier ou jeteur de sort-depuis l'Ancêtre jusqu'à Papa Longoué, le narrateur souligne la transmission d'un passé de résistance par le maintien de la mémoire des dominés qui, par ce biais, emprunte d'autres voies que les voies officielles de la transmission. C'est aussi pourquoi Glissant porte toute son attention sur des figures quotidiennes, inconnues, incarnation à la fois de la totalité et de personne, ce qui est une façon euphémisée de se distinguer de Césaire. Ce dernier évoquait davantage des grandes figures déterminantes de l'histoire antillaise comme Delgrès, Toussaint Louverture, etc. (Malela, Aimé Césaire). De la sorte, l'auteur de Malemort demeure dans l'historicité la plus proximale à l'instar de ses alliés objectifs comme Simone et André Schwarz-Bart dont les productions s'intéressent aussi à l'historicité à partir de figures quotidiennes, notamment la femme antillaise (Malela, "Discours littéraire "). On peut, dès lors, comprendre l'intention de Glissant lorsqu'il insiste sur le caractère pluriel avec "les " Longoué ou "les » Béluse, du fait qu'un Longoué ou un Béluse se fait l'écho des autres et, au-delà, du peuple des esclaves anonymes avec lesquels il y a une forme de parenté symbolique. 
En évoquant les différentes composantes des figures de l'univers antillais que l'auteur du Quatrième siècle perçoit, le récit s'inscrit d'emblée dans un monde proximal où sur le plan spatio-temporel l'éloigné devient proche par son caractère soudainement familier. Ainsi, la figure de l'Africain, figure moins édifiante qu'un Toussaint Louverture ou qu'un Delgrès, se constitue en archétype de la lutte contre l'aliénation culturelle, en l'occurrence la colonialité du pouvoir. Cet Africain symbolique sous le nom de Négateur, c'est-à-dire "l’Africain surpris dans sa terre et déporté sur la profondeur de mer et qui dès le premier jour avait refusé de se terrer dans le bas de ravine sous les fougères" (Glissant, Malemort 68), est celui-là même qui entre dans les représentations des protagonistes comme le " marron primordial " (68). Figure simple parce qu'il s'agit d'une mise en scène plus simplifiée de la complexité de l'histoire antillaise ; figure archétypale, parce que Glissant, part bien des principes (archai) pour expliquer l'origine (archè) de la présence au monde du sujet antillais. Autrement dit, il essaie de voir comment, à travers le marron et ses héritiers modernes, le passé et le présent sont rythmés par l'oubli de la temporalité singulière du marron :

Engloutis à l'abrupt de cette casse de cent cinquante années qui avait fêlé entre le premièrement innombrable d'antan et le deuxièmement trop dénombré présent, dans la même bavure de temps où la végétation de légende s'était amincie en savanes rêches, où les mots [et les significations] s'étaient pétrifiés comme d'une absence de quoi que ce soit à dire ou à désigner, où toute volonté de se souvenir de la première nuit et du Négateur s'était comme dessouchée des têtes et des ventres, où le mot mantou et le mot calloge le mot vezou-sans compter tant d'autres qui avaient vécu la vie raide des êtres clandestins menacés secrets-avaient peu à peu terni et disparu. (Malemort 69)

Si cet oubli accentué par le dessouchement a envahi les mémoires des protagonistes, il altère même le langage dont le lexique, pourtant imprégné de l'antan, aurait perdu de sa signification. Si cette perte touche certains protagonistes, elle n'interfère que rarement dans le folklore, qui forme l'âme de ces figures moins intimidantes. Dans cette reconfiguration, les personnages édifiants sont alors réinsérés dans un folklore où ils acquièrent une dimension moins grave, à la différence de ce qui aurait lieu dans les productions césairiennes. C’est le cas du général afro-caribéen de Cuba, Antonio Maceo (1845-96), dont un chant folklorique narre les aventures avec un effet tragicomique car " pâli incolore est un pur esprit un bon colon c'est Maceo général nègre de Cuba qu'on fit blanchir en effigie c'est Maceo " (184). Autre élément introduit dans le folklore est la narration ironique qui diffère la violence tout en installant celleci dans le langage. Le portrait du marron devient subitement éloigné parce qu'en Jamaïque il s'est fourvoyé dudit folklore, en internalisant la violence de la colonialité : "Jamaïque où se fit dans les Hauts une république de marrons qui bientôt traita de puissance à puissance avec les gouverneurs espagnols et bientôt traqua les apprentis marrons pour ensuite les remettre en bas aux colons chasseurs accompagnés des lourds dogues à colliers plombs » (186). 
Le retournement de la violence du marron ordinaire contre ses compatriotes souligne son caractère fragile, du fait que cette internalisation sera même incarnée par les quidams Beautemps et Tigamba qui, en tant que gendarme, demeure prêt à réprimer ses semblables (186-87). L'agent Tigamba a participé à la répression contre les grévistes, répression après laquelle il n’a pas été "inquiété ni poursuivi ; " de ce fait, il a pu continuer "à faire chaque matin sa tournée au marché aux poissons " (129-30). Ce sont là des petites gens par rapport auxquelles Toussaint Louverture apparaît lointain et abstrait, de telle sorte que l'on n'en sait plus rien " sinon que c'était un grand nègre [pour les Haïtiens qui] souquaient dur en soufflant, nus dans leur espérance, crachotant avec l'écume de leur bouche le nom Toussaint Toussaint " (178-79). La mise en scène de cette grande figure révolutionnaire dans le monde distal, ${ }^{1}$ par contraste avec les figures plus modestes, établit les différentes strates de la lutte contre la violence aliénante.

Cette même violence se retrouve également dans la conception historique du jeune Mathieu et du vieux Longoué, conception enracinée dans la temporalité et la spatial-

418 ité antillaise, d'où la répétition du langage de Longoué, " qui n'en avançait pas moins avec sûreté vers un savoir " (Quatrième siècle 14). C'est la clarification et l'agencement de cette connaissance que vise Mathieu (15), à savoir l'histoire avant l'incorporation ordonnée de la colonialité, avant la capture, puis la déportation ; bref, l'histoire avec laquelle sont venus Béluse et Longoué à travers la mer comme le rappelle ce monologue de Mathieu : "Et aucun de nous ne connaît ce qui s'est passé dans le pays là-bas au-delà des eaux, la mer a roulé sur nous tous, même toi qui vois l'histoire et les tenants " (59). Si, à travers la figure de Mathieu, Glissant insiste tant sur l'idée que les ancêtres de Mathieu et Papa Longoué ont compris rapidement la fin de l'histoire qui les liait au monde africain (qui est impliqué dans la terre perdue), c'est pour mieux souligner la modification du rapport à l'histoire que cette déportation a généré en créant d'abord un silence autour d'elle (33-34).

Ce silence se dresse devant Mathieu comme un obstacle à la présence antillaise au monde. Ceci implique de rompre le silence en explorant soi-même cette histoire qui a créé aussi une autre subjectivité. C’est ce qui incite Mathieu à vouloir " découvrir de l'intérieur, reprendre à partir du moment où tout n'était pas obscur, évanoui, à partir du dernier moment où nous avons pu regarder le soleil au-dessus de la Pointe des Sables, nous asseoir dans le bois d'acacias pour peser cette lumière et savoir qu'elle était en nous! » (58). Ce nouveau rapport à l'histoire ou cette expérience de l'histoire introduit une mutation profonde de la subjectivité africaine au contact de la société de plantation qui va rejeter dans le monde distal la terre d'Afrique à jamais perdue. De la sorte, Glissant s'inscrit encore en marge du discours césairien en reconnaissant effectivement l'aliénation de l'esclavage, mais en faisant de l'Afrique cette fois non pas une historicité déterminante jusqu'au point de devenir le moment propice pour la refondation de la subjectivité antillaise, mais une historicité déterminante seulement pour appréhender l'une des dimensions de la souffrance antillaise. Dans cette optique, l'on comprend pourquoi pour le narrateur, « [t]oute l'histoire s'éclaire dans la terre» (47). 
Si l'historicité des Antilles est appréciée par la référence à la terre du pays, il ne se trouve pas moins que la construction d'une telle historicité entre en tension avec ceux qui veulent l'assumer en raison de son caractère hélicoïdal ; mais la tentative de configuration apparaît comme linéaire, d'où la difficulté de faire dialoguer la logique du quimboiseur avec le questionnement de Mathieu : "Ne sachant pas encore que Mathieu l'avait vaincu, puisque le jeune homme le forçait à suivre le sentier 'du plus logique', et que voici qu'il raisonnait en que, en donc, en après et avant, avec des nœuds de pourquoi dans sa tête, noyés dans une tempête de parce que " (47). L'évocation de cette logique du quimboiseur permet à Glissant de demeurer dans son écriture immanente en mettant en avant d'abord la figure des colons comme La Roche et Senglis " solitaire dans son monde unique sans nuances ni doutes " (76); ensuite, la figure du marron Longoué " épicentre des marrons " qui vont lancer la révolte (94) devenu par la suite quimboiseur, face à la figure de l'esclave resté dans l'habitation (Béluse).

C'est donc à partir de l'expérience fondamentale de la parenté incarnée par les figures déterminantes de proximités que Glissant assume une mémoire du passé, c'est-à-dire déconstruit la part d'aliénation présente dans l'identité antillaise. Ou si l'on préfère, cette perspective proximale fondamentale opposée à l'idée de système, privilégie une approche concrète pour mettre en lumière le caractère complexe de la totalité dispersée et refuser le moment du rassemblement hégélien. Son écriture sera donc immanente, comme on l'a vu, à travers la promotion de la notion d'expérience déjà imprégnée d'une violence aliénante qu'il y a lieu de maîtriser. Cette aliénation est précisément la deuxième expérience protéiforme que souligne Glissant.

Il s'agit de porter une critique de l'aliénation, ce qui relève de l'usage de la généalogie, une démarche en quelque sorte nietzschéenne et que l'on retrouve aussi chez Foucault notamment dans son Archéologie du savoir (1969). Quant à Glissant, il met en scène les liens de parenté qui unissent et divisent le personnel littéraire. Ce recours à la parenté permet ainsi au narrateur d'assurer une mémoire du passé, c'està-dire de l'histoire, afin de montrer l'élection, parmi d'autres, de figures-celle de l'esclave et bien d'autres comme le marron, le quidam, le quimboiseur, l'Africain, etc. -incarnant bien la souffrance et la subjectivité. Bien plus, le lien de parenté permet à Glissant de donner à voir d'autres bases, à partir desquelles évoquer les Antilles pour les penser dans l'écart à la colonialité du pouvoir.

\section{Colonialité du POUVOIR OU DE LA Violence}

La violence traitée dans les deux productions glissantiennes apparaît à travers l'apprentissage forcé de la langue nouvelle. Celle-ci crée une forme de coupure avec le lieu d'origine et met l'esclave devant les conditions de son présent douloureux. Tel est le sort des nouveaux esclaves dans une plantation où ils doivent oublier " de se révolter pendant qu'ils apprennent la langue d'ici ; et quand ils connaissent la langue 
c'est trop tard, ils sont matés " (Quatrième siècle 83). Il s'agit donc d'une sorte de déracinement de ce que Glissant nomme "la souche " (83), d'un arrachement à la terre d'origine qui conduit à la perte patronymique. C'est pourquoi le marron Longoué éprouve quelques difficultés à communiquer avec Louise dont il finit par accepter la parole nouvelle sans rien céder de sa science d'Afrique, car " il était obligé de s'y mettre, s'il voulait communiquer avec les autres marrons. En revanche, ce n'était pas nécessaire qu'elle connût ses mots à lui ; et il n'était pas fâché d'assurer son prestige d'Africain, en partageant le moins possible sa science du pays d'au-delà les eaux " (95).

À cette violence par l'assimilation de la norme de l'aliénation (ou colonialité), s'ajoute, pour ces mêmes esclaves, la "damnation physique et terrifiante " (101) dans les habitations, damnation qui va plus loin encore si l'esclave venait à être arrêté après une tentative de marronnage (92). Devant cette violence à la fois symbolique et physique, la folie s'y ajoute en tant que lieu de manifestation de la différence et de la privation de soi-même (Derrida 267), du corps violé par sa dépossession, du fait que ce corps de l'esclave dépend totalement de la volonté du maître, dépositaire de la violence légitime. C'est ainsi que la femme de Senglis contrôle, régule la descendance de ses esclaves, comme celle de Béluse (Quatrième siècle 114). On est ainsi dans une sorte de folie métaphysique au fondement même de l'habitation de Senglis et c'est encore elle qui la précipite dans le déclin comme l'a bien compris Béluse :

Béluse comprit obscurément que l'habitation avait jusque-là vécu dans l'ombre de cette folie qui seule avait maintenu conjoints, par sa force surnaturelle de contagion ou d'hypnose [...], les univers, les divers secteurs dont il avait deviné, plus que cerné, les contours. La disparition de cette obsession compromit l'équilibre de l'ensemble, et dès lors ce fut le déclin. (115)

La folie métaphysique inscrite dans la différence affecte alors le personnel littéraire qui se trouve entraînée dans cette souffrance qui, dans la perspective de Glissant, demeure avant tout une expérience concrète ainsi qu'une des conditions de possibilité de la violence.

Si, dans Le Quatrième siècle, Glissant lie le problème de la conscience à celui de l'histoire en faisant du monde esclavagiste et de la figure du marron une valeur historiale contre la colonialité du pouvoir dans le discours antillais instituée, dans Malemort (1975), il revient sur cet aspect figuratif pour marquer différemment sa tendance à l'historicité. Celle-ci se construit comme un discours en contrepoint au discours fondé sur la violence épistémique (Spivak). C'est pourquoi l'écart épistémique que veut introduire Glissant fait la promotion d'une approche plus empirique qui fait voir la complexité du réel antillais. Dans cette optique, l'auteur de Malemort ajoute au dispositif abstrait qu'est l'histoire l'expérience de la violence incarnée notamment par les différentes figures quotidiennes qui sont mobilisées dans Malemort.

Et l'expérience de la violence telle qu'elle est donnée à voir concerne également la colonialité proprement dite, dans la mesure où il s'agit de fictionnaliser le processus par lequel la " male mort, " c'est-à-dire une mort par laquelle le dominé se voit dépos- 
sédé de son pays parce que dominé par l'extériorité, frappe l'ensemble du personnel littéraire. En effet, les structures de l'État colonial ont transmuté pour ne conserver que la forme abstraite de la colonialité du pouvoir. L'identité dite culturelle se trouve prise en charge par la colonialité (Glissant, Malemort 198) qui apparaît comme allant de soi, comme faisant partie du sens commun, puisque l'aliénation incorporée du système de représentation de la domination devient la mesure pour tout discours. De là, le discours du nouvel arrivant dans les Antilles prend une dimension de relais de celui des officiels dans toutes les formes de sa manifestation :

La théorie des arrivants visitants demeurants que par les mots détournés il t'expliqua et te rendit visible :

L'un précis, sobre, savant. Qui éclate en aigus de compréhension. Qui touche dru aux problèmes. Sa clé des choses devant lui comme un viseur. Il raie d'un trait les emberlificotés. Il porte la loi de la fraternité internationale. Faites place.

$[\ldots]$

Un autre entend garder pour lui. Garder quoi ? L'entrée. N'est-ce pas qu'il faut être d'abord accepté ? N'est-ce pas que c'est difficile ? N'est-ce pas que je suis accepté ? Vous voyez, il a dit oui. (214)

Cette violence par la prise en charge du discours exogène se retrouve également dans l'organisation du pouvoir des représentants locaux qui se fait selon les critères des pouvoirs dits centraux. Ainsi chaque élection est confisquée par un groupe dominant et puissant qui tente de mettre en place ses candidats, d'où la narration de scènes tragi-comiques sur ces candidats appelés par des numéros (Deux, Trois, Quatre, etc.) (80-81). Mais la course à l'élection correspond à une recherche de reconnaissance du pouvoir de l'État dont les protagonistes ne peuvent se passer. Ainsi, l'autre est en eux par l'internalisation de sa violence symbolique comme le rapporte un protagoniste dans Malemort :

Oreste aime Hermione qui aime Pyrrhus qui aime Andromaque. Le nègre hait le mulâtre qui déteste le béké qui abomine le Blanc de France. Au-dessus de tout, il y a Hector. $\mathrm{Au}$-dessus de tous il y a la mère patrie. C'est la patrie qui choisit et élit, selon son bon plaisir. Voici pourquoi messieurs, hormis le bon argent à bon droit récolté, nous nous battons pour nos mairies. C'est pour savoir qu'Hector nous a choisis. Nous, qui pouvons si peu pour lui, hors d'affirmer le droit qu'il a de nous reconnaître. Et c'est pourquoi messieurs nous sommes vifs à nous combattre. (87)

Cette recherche de reconnaissance de la patrie nécessite un combat vif pour parvenir à la visibilité qui va jusqu’au remplissage des urnes, au recours aux électeurs fictifs (90) et à d'autres tricheries.

Si la transformation de la colonialité passe effectivement par la transmutation de l'État anciennement colonial et nouvellement promu " mère patrie " dont on cherche la reconnaissance, c'est que l'école est passée par là. Elle apparaît comme une désorientation des consciences qui touche les intellectuels de sorte que ceux-ci sont amenés à célébrer la culture légitime (115), allant jusqu'à s'enfermer dans les normes classiques (à travers par exemple la dictée sur Salammbô de Flaubert). Ce qui est le cas 
du personnage appelé Québec, un professeur d'anglais faisant l'intellectuel devant ses élèves :

Il avait renom d'homme cultivé, comme ailleurs des gens sont réputés habiles aux affaires. La " culture " dans ce pays confère statut. Du temps qu'il avait été instituteur, avant d'obtenir son poste d'anglicisant au lycée, il avait connu sa première célébrité pour la manière et le ton, sans compter l'attitude, dans lesquels il attaquait sa dictée préférée:

"C'était à Mégara virgule faubourg de Carthage virgule dans les jardins d'Hamilcar point à la ligne. " Et Hamilcar et Mégara se statufiaient au sortir de sa bouche, constellés de pierreries où fulguraient le savoir, la transcendance lointaine insoupçonnée, le sens de ce raffinement qui passait loin au-dessus de nos têtes de petits nègres inconscients. Eux les nègres savants. (158)

Ces " nègres savants" se rendront compte plus tard de leur errance dans une assimilation responsable de leur éloignement de leur terre (170). Pour Glissant, l'historicité est donc marquée par la figure ambivalente du marron anonyme et opposée à la violence de la colonialité, et porteur de cette même violence qu'il a incorporée et qu'il retourne ensuite contre ses semblables. Aussi figure déterminante, ou si l'on préfère historiale, sans devenir une figure en soi, la violence qu'il porte se décline dans l'internalisation des schémas institutionnels du successeur de l'État colonial qui adoube maire, député, intellectuels, etc. Ces derniers sont l'exemple manifeste de la "male mort » qui gagne les Antilles par la dépossession progressive de la subjectivité antillaise et la diffusion et l'inculcation de cette dépossession à tout le corps social.

Cette colonialité du pouvoir atteste de la violence qui caractérise le sujet dominé. Ainsi le déracinement-perte patronymique, coupure avec le lieu d'origine-la violence physique, la folie métaphysique inscrite dans la différence, l'incorporation de la colonialité telle la prise en charge antillaise du discours exogène, la recherche de la reconnaissance de l'État colonial et la désorientation par l'école. Tout cet ensemble affecte le personnel littéraire qui se trouve pris dans cette souffrance généralisée que l'auteur pense comme expérience et condition de possibilité de la violence.

Ainsi le discours glissantien est axé sur la parenté et la violence. Cette dernière enrichit la perception des Antilles qui acquièrent une valeur historiale dans son discours.

\section{EN GUISE DE CONCLUSION}

Dans notre propos liminaire, nous avons mis en évidence l'importance de la problématique de de la colonialité (ou aliénation), de la subjectivité et de l'altérité, dans leur rapport à l'histoire des Antilles, à partir du cas d'Édouard Glissant. Dans cette perspective, notre but a été de montrer la manière dont cet écrivain et penseur antillais approche le global à un moment particulier de son historicité. Il se confronte au problème de la subjectivité en déconstruisant l'aliénation à partir d'une approche concrète qui aboutit à une autre forme d'ambivalence de son cadre discursif. Pour le 
montrer, ce propos s'est appuyé sur deux arguments de force : d'abord, la transformation du monde des idées autour du courant poststructuraliste qui a légitimé d'autres possibles favorables à une critique plus épistémologique de la colonialité du pouvoir ; ensuite, la mise en relief de figures historiales ainsi que la mobilisation de la violence constitutive de la subjectivité antillaise selon Glissant.

Le premier argument relatif à la transposition du champ des idées dans la production glissantienne montre qu'il s'agit d'une contestation précipitée par une nouvelle génération de penseurs réservés sur l'existentialisme, le structuralisme et la notion de système promue par Hegel. Elle a aussi mobilisé la notion de généalogie nietzschéenne. C'est dans cette optique que Glissant opte pour une approche concrète qui lui permet de conserver une relative proximité avec l'historicité et l'altérité et donc d'aborder l'expérience de l'aliénation, de la subjectivité et de l'altérité à travers l'évocation de l'esclavage et des figures historiales telle que le marron, incarnation de la violence.

De là, peut se déployer notre second argument autour des figures historiales et de l'expérience de la violence comme métaphores de la reconstruction de l'identité antillaise chez Glissant. En effet, ces figures peuvent incarner des liens de parenté dont la fonction principale est d'assurer une mémoire du passé. C'est pourquoi Glissant utilise une écriture immanente et bien ancrée dans la terre antillaise, ce qui caractériserait l'identité différée qu'il propose par rapport à l'aliénation et à la violence de celle-ci, violence qui est aussi bien physique que symbolique.

En évoquant à la fois les figures historiales et les expériences de la violence, Glissant aboutit à une autre forme d'ambivalence de son discours par la répétition de la parole de l'altérité et de la figuration des Antilles comme attraction et dérision. Ce résultat procède de l'approche concrète qui fait voir des détails qui eux-mêmes complexifient le rapport à la subjectivité et à l'autre. Dans cette optique, le rapport à l'aliénation et à l'altérité s'effectue d'une part après leur déconstruction par la mobilisation des expériences de la parenté et d'autre part par la reconstruction d'une subjectivité antillaise autre qui passe par la "Relation, " ce qui traduit la position politique de Glissant ainsi que celle du champ des idées. La subjectivité se trouve alors ancrée dans une historicité en changement perpétuel (metabolè) et en régénération permanente (genesis).

\section{Note}

1. Le monde éloigné ou mis à distance. 


\section{OUVRages CITÉS}

Besnier, Jean-Michel. Histoire de la philosophie moderne et contemporaine. Figures et cuvres. Grasset, 1993.

Bhabha, Homi K. Les lieux de la culture. Une théorie postcoloniale, traduit par François Bouillot, Payot, 2007.

Bourdieu, Pierre. Les règles de l'art. Genèse et structure du champ littéraire. Seuil, 1998.

Radford, Daniel. Édouard Glissant. Seghers, 1982.

Couloubaritsis, Lambros. "Événement et temps propice : Platon et Kazantzaki. " Que se passe-t-il ? Événements, sciences humaines et littérature, édité par Didier Alexandre, Madeleine Frédéric, Sabine Parent, et Michèle Touret, PU de Rennes, 2005, pp. 39-52.

---. Histoire de la philosophie ancienne et médiévale. Figures illustres. Grasset, 1998.

---. La Proximité et la question de la souffrance humaine. En quête de nouveaux rapports de l'homme avec soi-même, les autres, les choses et le monde. Ousia, 2005.

Derrida, Jacques. L'Écriture et la différence. Seuil, 1967.

Fonkoua, Romuald. Essai sur une mesure du monde au XXe siècle: Édouard Glissant. Honoré Champion, 2002.

Glissant, Édouard. L'Intention poétique. Seuil, 1969.

---. Malemort. Gallimard, 1975 (1997).

---. Le Quatrième siècle. Seuil, 1964.

Grosfoguel, Ramon. " Les implications des altérités épistémiques dans la redéfinition du capitalisme global : transmodernité, pensée frontalière et colonialité globale. " Multitudes, vol. 26, 2006, pp. 62-63.

Hottois, Gilbert. De la Renaissance à la Postmodernité. Une histoire de la philosophie moderne et contemporaine. De Boeck U, 2002.

Kuhn, Thomas. La Structure des révolutions scientifiques, traduit par Laure Meyer, Flammarion, 1983.

Malela, Buata. Aimé Césaire. Le fil et la trame. Critique et figurations de la colonialité du pouvoir. Anibwe, 2009.

---. " Discours littéraire et pensée féministe. De Simone de Beauvoir à Simone Schwarz-Bart. » Romanica Silesiana, vol. 8, no. 1, 2013, pp. 175-83.

---. "Les enjeux de la figuration de Lumumba. Débat postcolonial et discours en contrepoint chez Césaire et Sartre. " Mouvements, vol. 51, 2007, pp. 130-41.

Marx, Karl, et Friedrich Engels. Philosophie, édité par Maximilien Rubel, Gallimard, 1982. 
McDonald, Christie, et Susan Rubin Suleiman. French Global. Une nouvelle perspective sur l'histoire littéraire. Classiques Garnier, 2014.

Ménil, Alain. Les Voies de la créolisation, essai sur Édouard Glissant. De l'incidence éditeur, 2011.

Norvat, Manuel. Le chant du divers. Introduction à la philopoétique d'Édouard Glissant. L'Harmattan, 2015.

Saïd, Edward. Culture et Impérialisme, traduit par Paul Chemla, Fayard/Le Monde diplomatique, 2000.

Wald Lasowski, Aliocha. Édouard Glissant, penseur des archipels. Pocket, 2015. 